\title{
Solving of Klein-Gordon by Two Methods of Numerical Analysis
}

\author{
Joseph Bonazebi Yindoula ${ }^{1 *}$, Alphonse Massamba², Gabriel Bissanga ${ }^{1}$ \\ ${ }^{1}$ Laboratory of Numerical Analysis, Kibernetics and Applications, University Marien NGOUABI, Brazzaville, Congo \\ ${ }^{2}$ Division of Physics, Brazzaville Institute of Technology, Brazzaville, Congo \\ Email: ‘bonayindoula@yahoo.fr
}

How to cite this paper: Yindoula, J.B., Massamba, A. and Bissanga, G. (2016) Solving of Klein-Gordon by Two Methods of Numerical Analysis. Journal of Applied Mathematics and Physics, 4, 1916-1929.

http://dx.doi.org/10.4236/jamp.2016.410194

Received: August 15, 2016

Accepted: October 23, 2016

Published: October 27, 2016

Copyright $\odot 2016$ by authors and Scientific Research Publishing Inc. This work is licensed under the Creative Commons Attribution International License (CC BY 4.0)

http://creativecommons.org/licenses/by/4.0/

\begin{abstract}
In this paper, the Decomposion Laplace-Adomian method and He-Laplace method are used to construct the solution of Klein-Gordon equation.
\end{abstract}

\section{Keywords}

Laplace-Adomian Method, He-Laplace Method, Klein-Gordon Equation

\section{Introduction}

In field theory, the description of the free partide for the wave function in quantum physics obeys to Klein-Gordon equation [1]. In addition, it also appears in nonlinear optics and plasma physics.

In sum, the Klein-Gordon equation rises in physics in linear and non linear forms. In this paper we examine the Klein-Gordon equation, using the Laplace-Adomian decomposition method and He-Laplace method to get the exact solution. The KleinGordon equation is described as:

$$
u_{t t}(x, t)=u_{x x}(x, t)+\alpha u(x, t)+\beta N(u(x, t))+h(x, t)
$$

where $\alpha, \beta$ are constants (spin zero) charged field, $h(x, t)$ is a source term and $N(u(x, t))$ is a nonlinear function of $u(x, t)$.

\section{Describing of Both Method}

\subsection{The Laplace Transform [2]}

Let's note the laplace transform by

$$
U(x, s)=(u(x, t))=\int_{0}^{\infty} u(x, t) \mathrm{e}^{-s t} \mathrm{~d} t
$$


From (1), we have:

$$
\left\{\begin{array}{l}
\left(\frac{\partial u(x, t)}{\partial t}\right)=\int_{0}^{\infty} \mathrm{e}^{-s t} \frac{\partial u}{\partial t} \mathrm{~d} t=s(u(x, t))-u(x, 0) \\
\left(\frac{\partial^{2} u(x, t)}{\partial t^{2}}\right)=s^{2}(u(x, t))-s u(x, 0)-\frac{\partial u(x, 0)}{\partial t} \\
\left(\frac{\partial u(x, t)}{\partial x}\right)=\int_{0}^{\infty} \mathrm{e}^{-s t} \frac{\partial u}{\partial x} \mathrm{~d} t=\frac{\mathrm{d} U}{\mathrm{~d} x} \\
\left(\frac{\partial^{2} u(x, t)}{\partial x^{2}}\right)=\frac{\mathrm{d}^{2} U}{\mathrm{~d} x^{2}}
\end{array}\right.
$$

\subsection{Laplace-Adomian Decomposition Method (LADM) [3]-[6]}

Suppose that we need to solve the following equation:

$$
u_{t t}(x, t)-u_{x x}(x, t)+\alpha u(x, t)+\beta N(u(x, t))=h(x, t)
$$

subject to initial conditions:

$$
u(x, 0)=f(x) ; u_{t}(x, 0)=g(x)
$$

$E$ is a Banach space, where $F: E \rightarrow E$ is a linear or a nonlinear operator, $h \in E$ and $u$ is the unknown function.

Let's suppose that operator $F$ can be decomposed under the following form:

$$
F=L+R+N
$$

where $L+R$ is linear, $N$ nonlinear. Let's suppose that $L$ is inversible to the sense of Adomian with $L^{-1}$ as inverse.

From above, by applying the Laplace transform to both sides of Equation (4), we have:

$$
\left[u_{t t}(x, t)\right]-\left[u_{x x}(x, t)-\alpha u(x, t)\right]+\beta[N(u(x, t))]=(h(x, t))
$$

From the Equation (7), it follows:

$$
s^{2}(u(x, t))-s u(x, 0)-u_{t}(x, 0)-\left[u_{x x}(x, t)-\alpha u(x, t)\right]+\beta[N(u(x, t))]=(h(x, t))(8)
$$

and this equation gives

$$
s^{2}(u(x, t))=s u(x, 0)+u_{t}(x, 0)+(h(x, t))+\left[u_{x x}(x, t)-\alpha u(x, t)\right]-\beta[N(u(x, t))]
$$

So, from the above Equation (9), we can write:

$$
\begin{aligned}
(u(x, t))=\frac{1}{s} u(x, 0)+\frac{1}{s^{2}} & u_{t}(x, 0)+\frac{1}{s^{2}}(h(x, t))+\frac{1}{s^{2}}\left[u_{x x}(x, t)-\alpha u(x, t)\right]-\frac{1}{s^{2}} \beta[N(u(x, t))] \\
\text { We have now } u(x, t): & \\
u(x, t)= & \mathcal{L}^{-1}\left(\frac{1}{s} f(x)\right)+\mathcal{L}^{-1}\left(\frac{1}{s^{2}} g(x)\right)+\mathcal{L}^{-1}\left(\frac{1}{s^{2}} \mathcal{L}(h(x, t))\right) \\
& +\mathcal{L}^{-1}\left(\frac{1}{s^{2}} \mathcal{L}\left[u_{x x}(x, t)-\alpha u(x, t)\right]\right)-\mathcal{L}^{-1}\left(\frac{1}{s^{2}} \beta \mathcal{L}[N(u(x, t))]\right)
\end{aligned}
$$


We research solution of (4) in the following series expansion form

$$
u(x, t)=\sum_{n=0}^{+\infty} u_{n}(x, t)
$$

and we consider

$$
N u(x, t)=\sum_{n=0}^{+\infty} A_{n}
$$

where $A_{n}$ are the Adomian polynomials of $u_{0}, u_{1}, \cdots, u_{n}$ and it can be calculated by formula given below.

$$
A_{n}=\frac{1}{n !} \frac{\mathrm{d}^{n}}{\mathrm{~d} p^{n}}\left[N \sum_{i=0}^{\infty} \lambda^{i} u_{i}\right]_{\lambda=0}, n=0,1,2,3, \cdots
$$

Using Equation (12) and Equation (13) in Equation (11) we have:

$$
\begin{aligned}
\sum_{n=0}^{+\infty} u_{n}(x, t)= & \mathcal{L}^{-1}\left(\frac{1}{s} f(x)\right)+\mathcal{L}^{-1}\left(\frac{1}{s^{2}} g(x)\right)+\mathcal{L}^{-1}\left(\frac{1}{s^{2}} \mathcal{L}(h(x, t))\right) \\
& +\mathcal{L}^{-1}\left(\frac{1}{s^{2}} \mathcal{L}\left[\sum_{n=0}^{+\infty}\left(\frac{\partial^{2}\left(u_{n}(x, t)\right)}{\partial x^{2}}\right)-\alpha \sum_{n=0}^{+\infty} u_{n}(x, t)\right]\right)-\mathcal{L}^{-1}\left(\frac{1}{s^{2}} \beta \mathcal{L}\left(\sum_{n=0}^{+\infty} A_{n}\right)\right)
\end{aligned}
$$

From (15), we have the following Adomian algorithm:

$$
\left\{\begin{array}{l}
u_{0}(x, t)=\mathcal{L}^{-1}\left(\frac{1}{s} f(x)\right)+\mathcal{L}^{-1}\left(\frac{1}{s^{2}} g(x)\right)+\mathcal{L}^{-1}\left(\frac{1}{s^{2}} \mathcal{L}(h(x, t))\right)=K(x, t) \\
u_{n+1}(x, t)=\mathcal{L}^{-1}\left(\frac{1}{s^{2}} \mathcal{L}\left[\left(\frac{\partial^{2}\left(u_{n}(x, t)\right)}{\partial x^{2}}\right)-\alpha u_{n}(x, t)\right]\right)-\mathcal{L}^{-1}\left(\frac{1}{s^{2}} \beta \mathcal{L}\left(A_{n}\right)\right), n \geq 0
\end{array}\right.
$$

and we obtain the Adomian algorithm:

$$
\left\{\begin{array}{l}
u_{0}(x, t)=K(x, t) \\
u_{n+1}(x, t)=\mathcal{L}^{-1}\left(\frac{1}{s^{2}} \mathcal{L}\left[\left(\frac{\partial^{2}\left(u_{n}(x, t)\right)}{\partial x^{2}}\right)-\alpha u_{n}(x, t)\right]\right)-\mathcal{L}^{-1}\left(\frac{1}{s^{2}} \beta \mathcal{L}\left(A_{n}\right)\right), n \geq 0
\end{array}\right.
$$

\section{Remark}

In order overcome the short coming, we assume that $K(x, t)$ can be divided into the sum of two parts namely $K_{0}(x, t)$ and $K_{1}(x, t)$. Therefore,we get:

$$
K(x, t)=K_{0}(x, t)+K_{1}(x, t)
$$

Instead of the iteration procedure Equation (17) we suggest the following modification

$$
\left\{\begin{array}{l}
u_{0}(x, t)=K_{0}(x, t) \\
u_{1}(x, t)=K_{1}(x, t)+\mathcal{L}^{-1}\left(\frac{1}{s^{2}} \mathcal{L}\left[\left(\frac{\partial^{2}\left(u_{0}(x, t)\right)}{\partial x^{2}}\right)-\alpha u_{0}(x, t)\right]\right)-\mathcal{L}^{-1}\left(\frac{1}{s^{2}} \beta \mathcal{L}\left(A_{0}\right)\right) \\
u_{n+1}(x, t)=\mathcal{L}^{-1}\left(\frac{1}{s^{2}} \mathcal{L}\left[\left(\frac{\partial^{2}\left(u_{n}(x, t)\right)}{\partial x^{2}}\right)-\alpha u_{n}(x, t)\right]\right)-\mathcal{L}^{-1}\left(\frac{1}{s^{2}} \beta \mathcal{L}\left(A_{n}\right)\right), n \geq 1
\end{array}\right.
$$


The solution through the modified Laplace decomposition method highly depends upon the choice of $K_{0}(x, t)$ and $K_{1}(x, t)$.

\subsection{He-Laplace Method [7]}

We consider a general nonlinear non homogeneous partial differential equation with initial conditions of the form

$$
\left\{\begin{array}{l}
u_{t t}(x, t)-u_{x x}(x, t)+\alpha u(x, t)+\beta N(u(x, t))=h(x, t) \\
u(x, 0)=f(x) \\
u_{t}(x, 0)=g(x)
\end{array}\right.
$$

$N$ represents the general nonlinear differential operateur and $h(x, t)$ is the source term.

Taking the Laplace transform on both sides of (20), we obtain:

$$
\begin{aligned}
& \mathcal{L}\left[u_{t t}(x, t)\right]-\mathcal{L}\left[u_{x x}(x, t)-\alpha u(x, t)\right]+\beta \mathcal{L}[N(u(x, t))]=\mathcal{L}(h(x, t)) \\
\Leftrightarrow & \\
& s^{2}(u(x, t))=s u(x, 0)+u_{t}(x, 0)+(h(x, t))+\left[u_{x x}(x, t)-\alpha u(x, t)\right]-\beta[N(u(x, t))]
\end{aligned}
$$

Applying the initial conditions given in (22), we have:

$$
\mathcal{L}(u(x, t))=\frac{1}{s} f(x)+\frac{1}{s^{2}} g(x)+\frac{1}{s^{2}} \mathcal{L}(h(x, t))+\frac{1}{s^{2}} \mathcal{L}\left[u_{x x}(x, t)-\alpha u(x, t)\right]-\frac{1}{s^{2}} \beta \mathcal{L}[N(u(x, t))]
$$

Operating the inverse Laplace transform on both sides of (23), we have

$$
\begin{aligned}
u(x, t)= & \mathcal{L}^{-1}\left(\frac{1}{s} f(x)\right)+\mathcal{L}^{-1}\left(\frac{1}{s^{2}} g(x)\right)+\mathcal{L}^{-1}\left(\frac{1}{s^{2}} \mathcal{L}(h(x, t))\right) \\
& +\mathcal{L}^{-1}\left(\frac{1}{s^{2}} \mathcal{L}\left[u_{x x}(x, t)-\alpha u(x, t)\right]\right)-\mathcal{L}^{-1}\left(\frac{1}{s^{2}} \beta \mathcal{L}[N(u(x, t))]\right)
\end{aligned}
$$

Now, we apply the homotopy perturbation method

$$
u(x, t)=\sum_{n=0}^{\infty} p^{n} u_{n}(x, t)
$$

and the non linear term can be decomposed as

$$
N u(x, t)=\sum_{n=0}^{\infty} p^{n} H_{n}(u)
$$

for some He's polynomials $H_{n}(u)$ that are given by

$$
H_{n}(u)=\frac{1}{n !} \frac{\partial^{n}}{\partial p^{n}}\left[N \sum_{n=0}^{\infty} p^{i} u_{i}\right]_{p=0}, n=0,1,2,3, \cdots
$$

Sustituding Equation (25) and Equation (26) in Equation (24), we get

$$
\begin{aligned}
\sum_{n=0}^{\infty} p^{n} u_{n}(x, t)= & \mathcal{L}^{-1}\left(\frac{1}{s} f(x)\right)+\mathcal{L}^{-1}\left(\frac{1}{s^{2}} g(x)\right)+\mathcal{L}^{-1}\left(\frac{1}{s^{2}}(h(x, t))\right) \\
& +p\left(\sum_{n=0}^{\infty} \mathcal{L}^{-1}\left(\frac{1}{s^{2}} \mathcal{L}\left[\frac{\partial^{2}\left(p^{n} u_{n}(x, t)\right)}{\partial x^{2}}-\alpha p^{n} u_{n}(x, t)\right]\right)-\sum_{n=0}^{\infty} \beta \mathcal{L}^{-1}\left(\frac{1}{s^{2}} \mathcal{L}\left[p^{n} H_{n}(u)\right]\right)\right)
\end{aligned}
$$


Comparing the coefficients of like powers of $p$, we have the following approximations:

$$
\left\{\begin{aligned}
p^{0}: u_{0}(x, t) & =\mathcal{L}^{-1}\left(\frac{1}{s} f(x)\right)+\mathcal{L}^{-1}\left(\frac{1}{s^{2}} g(x)\right)+\mathcal{L}^{-1}\left(\frac{1}{s^{2}} \mathcal{L}(h(x, t))\right) \\
p^{1}: u_{1}(x, t) & =\mathcal{L}^{-1}\left(\frac{1}{s^{2}} \mathcal{L}\left[\frac{\partial^{2}\left(u_{0}(x, t)\right)}{\partial x^{2}}-\alpha u_{0}(x, t)\right]\right)-\beta \mathcal{L}^{-1}\left(\frac{1}{s^{2}} \mathcal{L}\left[H_{0}(u)\right]\right) \\
p^{2}: u_{2}(x, t) & =\mathcal{L}^{-1}\left(\frac{1}{s^{2}} \mathcal{L}\left[\frac{\partial^{2}\left(u_{1}(x, t)\right)}{\partial x^{2}}-\alpha u_{1}(x, t)\right]\right)-\beta \mathcal{L}^{-1}\left(\frac{1}{s^{2}} \mathcal{L}\left[H_{1}(u)\right]\right) \\
\vdots &
\end{aligned}\right.
$$

\section{Illustrative Examples}

To demonstrate the applicability of the above-presented method, we have applied it to two linear and two non linear partial differential equations. These examples have been chosen because they have been widely discussed in literature.

\subsection{Example 1}

Consider the following linear Klein-Gordon equation

$$
\left\{\begin{array}{l}
u_{t t}(x, t)-u_{x x}(x, t)-u(x, t)=-\cos x \sin t \\
u(x, 0)=0 \\
u_{t}(x, 0)=\cos x
\end{array}\right.
$$

\subsubsection{Application of the LADM}

Applying the Laplace transform on both side of Equation (30) with the initial conditions, we have:

$$
\mathcal{L}(u(x, t))=\frac{1}{s^{2}} \cos x-\left(\frac{1}{s^{2}\left(s^{2}+1\right)}\right) \cos x+\frac{1}{s^{2}} \mathcal{L}\left[u_{x x}(x, t)+u(x, t)\right]
$$

The inverse Laplace transform give us:

$$
u(x, t)=\mathcal{L}^{-1}\left(\frac{1}{s^{2}}\right) \cos x-\mathcal{L}^{-1}\left(\frac{1}{s^{2}\left(s^{2}+1\right)}\right) \cos x+\mathcal{L}^{-1}\left(\frac{1}{s^{2}} \mathcal{L}\left(u_{x x}(x, t)+u(x, t)\right)\right)
$$

$\Leftrightarrow$

$$
u(x, t)=\sin t \cos x+\mathcal{L}^{-1}\left(\frac{1}{s^{2}}\left(u_{x x}(x, t)+u(x, t)\right)\right)
$$

We suppose that solution of (30) has the following form:

$$
u(x, t)=\sum_{n=0}^{+\infty} u_{n}(x, t)
$$

From (34) and (33). we have:

$$
\sum_{n=0}^{+\infty} u_{n}(x, t)=\sin t \cos x+\sum_{n=0}^{+\infty} \mathcal{L}^{-1}\left(\frac{1}{s^{2}} \mathcal{L}\left(\frac{\partial^{2}\left(u_{n}(x, t)\right)}{\partial x^{2}}+u_{n}(x, t)\right)\right)
$$


This result garantee that the following Adomian algorithm is:

$$
\left\{\begin{array}{l}
u_{0}(x, t)=\sin t \cos x \\
u_{n+1}(x, t)=\mathcal{L}^{-1}\left(\frac{1}{s^{2}} \mathcal{L}\left(\frac{\partial^{2}\left(u_{n}(x, t)\right)}{\partial x^{2}}+u_{n}(x, t)\right)\right), \forall n \geq 0
\end{array}\right.
$$

Consequently,we obtain:

$$
\left\{\begin{array}{l}
u_{0}(x, t)=\sin t \cos x \\
u_{n}(x, t)=0, \forall n \geq 1
\end{array}\right.
$$

So that the solution of (30) is given by

$$
u(x, t)=\sum_{n=0}^{+\infty} u_{n}(x, t)=u_{0}(x, t)=\sin t \cos x
$$

which is the exact solution of problem.

\subsubsection{Application of the He-Laplace Method}

Applying the Laplace transform on both side of Equation (30) with the initial conditions, we obtain:

$$
\mathcal{L}(u(x, t))=\frac{1}{s^{2}} \cos x-\left(\frac{1}{s^{2}\left(s^{2}+1\right)}\right) \cos x+\frac{1}{s^{2}} \mathcal{L}\left[u_{x x}(x, t)+u(x, t)\right]
$$

By applying inverse Laplace transform, we have:

$$
\begin{gathered}
u(x, t)=\mathcal{L}^{-1}\left(\frac{1}{s^{2}}\right) \cos x-\mathcal{L}^{-1}\left(\frac{1}{s^{2}\left(s^{2}+1\right)}\right) \cos x+\mathcal{L}^{-1}\left(\frac{1}{s^{2}} \mathcal{L}\left(u_{x x}(x, t)+u(x, t)\right)\right) \\
u(x, t)=\sin t \cos x+\mathcal{L}^{-1}\left(\frac{1}{s^{2}}\left(u_{x x}(x, t)+u(x, t)\right)\right)
\end{gathered}
$$

Now applying the homotopy perturbation method, we have:

$$
\sum_{n=0}^{\infty} p^{n} u_{n}(x, t)=\sin t \cos x+p \sum_{n=0}^{\infty} \mathcal{L}^{-1}\left(\frac{1}{s^{2}} \mathcal{L}\left[p^{n}\left(\frac{\partial^{2}}{\partial x^{2}}\left(u_{n}(x, t)\right)+u_{n}(x, t)\right)\right]\right)
$$

Comparing the coefficient of like powers of $p$, we have

$$
\left\{\begin{aligned}
p^{0}: u_{0}(x, t) & =\sin t \cos x \\
p^{1}: u_{1}(x, t) & =\mathcal{L}^{-1}\left(\frac{1}{s^{2}} \mathcal{L}\left[\left(\frac{\partial^{2}}{\partial x^{2}}\left(u_{0}(x, t)\right)+u_{0}(x, t)\right)\right]\right) \\
p^{2}: u_{2}(x, t) & =\mathcal{L}^{-1}\left(\frac{1}{s^{2}} \mathcal{L}\left[\left(\frac{\partial^{2}}{\partial x^{2}}\left(u_{1}(x, t)\right)+u_{1}(x, t)\right)\right]\right) \\
\vdots & \\
p^{n}: u_{n}(x, t) & =\mathcal{L}^{-1}\left(\frac{1}{s^{2}} \mathcal{L}\left[\left(\frac{\partial^{2}}{\partial x^{2}}\left(u_{n-1}(x, t)\right)+u_{n-1}(x, t)\right)\right]\right)
\end{aligned}\right.
$$

which gives us 


$$
\left\{\begin{array}{l}
p^{0}: u_{0}(x, t)=\sin t \cos x \\
p^{n}: u_{n}(x, t)=0, \forall n \geq 1
\end{array}\right.
$$

So that, the solution $u(x, t)$ is given by:

$$
u(x, t)=\lim _{p \rightarrow 1}\left(\sum_{n=0}^{\infty} p^{n} u_{n}(x, t)\right)=u_{0}(x, t)=\sin t \cos x
$$

\subsection{Exemple 2}

Consider the following nonlinear Klein-Gordon equation

$$
\left\{\begin{array}{l}
u_{t t}(x, t)-u_{x x}(x, t)+N(u(x, t))=2 x+t^{4} \\
u(x, 0)=0 \\
u_{t}(x, 0)=2
\end{array}\right.
$$

where $N(u)=\left[u_{x}(x, t)\right]^{2}$.

\subsubsection{Laplace-Adomian Method}

Using the Laplace transform, we have

$$
\begin{array}{cc} 
& s^{2} \mathcal{L}(u(x, t))-s u(x, 0)-\frac{\partial u(x, 0)}{\partial t}=\frac{2}{s} x+\frac{24}{s^{5}}+\mathcal{L}\left(\frac{\partial^{2} u(x, t)}{\partial x^{2}}\right)-\mathcal{L}\left(\left[\frac{\partial u(x, t)}{\partial x}\right]^{2}\right) \\
\Leftrightarrow & \mathcal{L}(u(x, t))=\frac{2}{s^{2}}+\frac{2}{s^{3}} x+\frac{24}{s^{7}}+\frac{1}{s^{2}} \mathcal{L}\left(\frac{\partial^{2} u(x, t)}{\partial x^{2}}\right)-\frac{1}{s^{2}} \mathcal{L}\left(\left[\frac{\partial u(x, t)}{\partial x}\right]^{2}\right)
\end{array}
$$

by applying inverse Laplace transformation to Equation (48), we hace

$$
u(x, t)=2 t+x t^{2}+\frac{1}{30} t^{6}+\mathcal{L}^{-1}\left(\frac{1}{s^{2}} \mathcal{L}\left(\frac{\partial^{2} u(x, t)}{\partial x^{2}}\right)\right)-\mathcal{L}^{-1}\left(\frac{1}{s^{2}} \mathcal{L}\left(\left[\frac{\partial u(x, t)}{\partial x}\right]^{2}\right)\right)
$$

Supposing that the solution of (46) has the following form:

$$
u(x, t)=\sum_{n=0}^{+\infty} u_{n}(x, t)
$$

and

$$
N u(x, t)=\sum_{n=0}^{+\infty} A_{n}
$$

Taking (50) and (51) in to (49), we obtain:

$$
\sum_{n=0}^{+\infty} u_{n}(x, t)=2 t+x t^{2}+\frac{1}{30} t^{6}+\sum_{n=0}^{+\infty}\left(\mathcal{L}^{-1}\left(\frac{1}{s^{2}} \mathcal{L}\left(\frac{\partial^{2} u_{n}(x, t)}{\partial x^{2}}\right)\right)-\mathcal{L}^{-1}\left(\frac{1}{s^{2}} \mathcal{L}\left(A_{n}\right)\right)\right)
$$

According to the standard Adomian algorithm (52), we need to chose $u_{0}(x, t)=2 t+x t^{2}+\frac{1}{30} t^{6}$. Here, we choose by convenience $u_{0}(x, t)=2 t+x t^{2}$. So, we have the following Adomian algorithm 


$$
\left\{\begin{array}{l}
u_{0}(x, t)=x t^{2}+2 t \\
u_{1}(x, t)=\frac{1}{30} t^{6}+\mathcal{L}^{-1}\left(\frac{1}{s^{2}} \mathcal{L}\left(\frac{\partial^{2} u_{0}(x, t)}{\partial x^{2}}\right)\right)-\mathcal{L}^{-1}\left(\frac{1}{s^{2}} \mathcal{L}\left(A_{0}\right)\right) \\
u_{n+1}(x, t)=\mathcal{L}^{-1}\left(\frac{1}{s^{2}} \mathcal{L}\left(\frac{\partial^{2} u_{n}(x, t)}{\partial x^{2}}\right)\right)-\mathcal{L}^{-1}\left(\frac{1}{s^{2}} \mathcal{L}\left(A_{n}\right)\right)
\end{array}\right.
$$

then garantee that:

$$
\left\{\begin{array}{l}
u_{0}(x, t)=x t^{2}+2 t \\
A_{0}=N\left(u_{0}(x, t)\right)=t^{4} \\
u_{1}(x, t)=\frac{1}{30} t^{6}-\frac{1}{30} t^{6}=0 \\
A_{n}=0, \forall n \geq 1 \Rightarrow u_{n}=0, \forall n \geq 1
\end{array}\right.
$$

So the exact solution of (46) is

$$
u(x, t)=x t^{2}+2 t
$$

\subsubsection{He-Laplace Method}

Using the Laplace transform, we have:

$$
\mathcal{L}(u(x, t))=\frac{2}{s^{2}}+\frac{2}{s^{3}} x+\frac{24}{s^{7}}+\frac{1}{s^{2}} \mathcal{L}\left(\frac{\partial^{2} u(x, t)}{\partial x^{2}}\right)-\frac{1}{s^{2}} \mathcal{L}\left(\left[\frac{\partial u(x, t)}{\partial x}\right]^{2}\right)
$$

Now, we apply the inverse Laplace transformation to Equation (46), we have:

$$
u(x, t)=2 t+x t^{2}+\frac{1}{30} t^{6}+\mathcal{L}^{-1}\left(\frac{1}{s^{2}} \mathcal{L}\left(\frac{\partial^{2} u(x, t)}{\partial x^{2}}\right)\right)-\mathcal{L}^{-1}\left(\frac{1}{s^{2}} \mathcal{L}\left(\left[\frac{\partial u(x, t)}{\partial x}\right]^{2}\right)\right)
$$

Applying the homotopy perturbation method, we have:

$$
\sum_{n=0}^{+\infty} p^{n} u_{n}(x, t)=2 t+x t^{2}+\frac{1}{30} t^{6}+p^{n+1} \sum_{n=0}^{+\infty}\left(\mathcal{L}^{-1}\left(\frac{1}{s^{2}} \mathcal{L}\left(\frac{\partial^{2} u_{n}(x, t)}{\partial x^{2}}\right)\right)-\mathcal{L}^{-1}\left(\frac{1}{s^{2}} \mathcal{L}\left(H_{n}(u)\right)\right)\right)
$$

where $H_{n}(u)$ are He's polynomials. The first few components of He's polynomials are given by

$$
\left\{\begin{array}{l}
H_{0}(u)=\left(\frac{\partial u_{0}(x, t)}{\partial x}\right)^{2}=t^{4} \\
H_{1}(u)=2\left(\frac{\partial u_{0}(x, t)}{\partial x}\right)\left(\frac{\partial u_{1}(x, t)}{\partial x}\right)=0 \\
H_{2}(u)=\left(\frac{\partial u_{1}(x, t)}{\partial x}\right)^{2}+2\left(\frac{\partial u_{0}(x, t)}{\partial x}\right)\left(\frac{\partial u_{2}(x, t)}{\partial x}\right)=0 \\
\vdots
\end{array}\right.
$$

Comparing the coefficients of the like powers of $p$, we have:

$$
p^{0}: u_{0}(x, t)=2 t+x t^{2}+\frac{1}{30} t^{6}
$$




$$
\begin{gathered}
p^{1}: u_{1}(x, t)=\mathcal{L}^{-1}\left(\frac{1}{s^{2}} \mathcal{L}\left(\frac{\partial^{2} u_{0}(x, t)}{\partial x^{2}}\right)\right)-\mathcal{L}^{-1}\left(\frac{1}{s^{2}} \mathcal{L}\left(H_{0}(u)\right)\right)=-\frac{1}{30} t^{6} \\
p^{n}: u_{n}(x, t)=\mathcal{L}^{-1}\left(\frac{1}{s^{2}} \mathcal{L}\left(\frac{\partial^{2} u_{n-1}(x, t)}{\partial x^{2}}\right)\right)-\mathcal{L}^{-1}\left(\frac{1}{s^{2}} \mathcal{L}\left(H_{n-1}(u)\right)\right)=0, \forall n \geq 2
\end{gathered}
$$

So that, the exact solution $u(x, t)$ is given by:

$$
\begin{aligned}
u(x, t) & =\lim _{p \rightarrow 1}\left(\sum_{n=0}^{\infty} p^{n} u_{n}(x, t)\right) \\
& =u_{0}(x, t)+u_{1}(x, t)+u_{2}(x, t)+u_{3}(x, t)+\cdots \\
& =2 t+x t^{2}+\frac{1}{30} t^{6}-\frac{1}{30} t^{6}+0+0+\cdots \\
& =2 t+x t^{2}
\end{aligned}
$$

\section{Applications}

\subsection{Problem 1}

Consider the following linear Klein-Gordon equation

$$
\left\{\begin{array}{l}
\frac{\partial^{2} u(x, t)}{\partial t^{2}}=\frac{\partial^{2} u(x, t)}{\partial x^{2}}+3 u(x, t)-3 \sin x \sin t \\
u(x, 0)=0 \\
u_{t}(x, 0)=\sin x
\end{array}\right.
$$

\section{Application of the LADM}

Using the Laplace transform, we have

$$
\begin{gathered}
s^{2}(u(x, t))-s u(x, 0)-\frac{\partial u(x, 0)}{\partial t}=\left(\frac{\partial^{2} u(x, t)}{\partial x^{2}}+3 u(x, t)\right)-3 \sin x(\sin t) \\
\Leftrightarrow \\
\mathcal{L}(u(x, t))=\frac{1}{s} u(x, 0)+\frac{1}{s^{2}} \frac{\partial u(x, 0)}{\partial t}-3\left(\frac{1}{s^{2}}\right)\left(\frac{1}{s^{2}+1}\right) \sin x+\frac{1}{s^{2}} \mathcal{L}\left(\frac{\partial^{2} u(x, t)}{\partial x^{2}}+3 u(x, t)\right)
\end{gathered}
$$

By appling the inverse Laplace transform, we have:

$$
\begin{aligned}
u(x, t)= & \mathcal{L}^{-1}\left(\frac{1}{s} u(x, 0)\right)+\mathcal{L}^{-1}\left(\frac{1}{s^{2}} \frac{\partial u(x, 0)}{\partial t}\right)-(3 \sin x) \mathcal{L}^{-1}\left(\frac{1}{s^{2}\left(s^{2}+1\right)}\right) \\
& +\mathcal{L}^{-1}\left(\frac{1}{s^{2}} \mathcal{L}\left(\frac{\partial^{2} u(x, t)}{\partial x^{2}}+3 u(x, t)\right)\right) \\
\Leftrightarrow & \\
u(x, t)=(\sin x) \mathcal{L}^{-1}\left(\frac{1}{s^{2}}\right)-(3 \sin x) \mathcal{L}^{-1}\left(\frac{1}{s^{2}\left(s^{2}+1\right)}\right)+\mathcal{L}^{-1}\left(\frac{1}{s^{2}} \mathcal{L}\left(\frac{\partial^{2} u(x, t)}{\partial x^{2}}+3 u(x, t)\right)\right) &
\end{aligned}
$$




$$
u(x, t)=3 \sin t \sin x-2 t \sin x+\mathcal{L}^{-1}\left(\frac{1}{s^{2}}\left(\frac{\partial^{2} u(x, t)}{\partial x^{2}}+3 u(x, t)\right)\right)
$$

From above equation, we have the following modified Adomian allgorithm:

$$
\left\{\begin{array}{l}
u_{0}(x, t)=\sin t \sin x \\
u_{1}(x, t)=2 \sin t \sin x-2 t \sin x+\mathcal{L}^{-1}\left(\frac{1}{s^{2}} \mathcal{L}\left(\frac{\partial^{2} u_{0}(x, t)}{\partial x^{2}}+3 u_{0}(x, t)\right)\right) \\
u_{n+1}(x, t)=\mathcal{L}^{-1}\left(\frac{1}{s^{2}} \mathcal{L}\left(\frac{\partial^{2} u_{n}(x, t)}{\partial x^{2}}+3 u_{n}(x, t)\right)\right), \forall n \geq 1
\end{array}\right.
$$

Equation (69) give us:

$$
\begin{aligned}
u_{1}(x, t) & =2 \sin t \sin x-2 t \sin x+\mathcal{L}^{-1}\left(\frac{1}{s^{2}} \mathcal{L}\left(\frac{\partial^{2} u_{0}(x, t)}{\partial x^{2}}+3 u_{0}(x, t)\right)\right) \\
& =2 \sin t \sin x-2 t \sin x+\mathcal{L}^{-1}\left(\frac{1}{s^{2}} \mathcal{L}(2 \sin t \sin x)\right) \\
& =2 \sin t \sin x-2 t \sin x+(2 \sin x) \mathcal{L}^{-1}\left(\frac{1}{s^{2}} \mathcal{L}(\sin t)\right) \\
& =2 \sin t \sin x-2 t \sin x+2 t \sin x-2 \sin x \sin t=0
\end{aligned}
$$

Thus

$$
\left\{\begin{array}{l}
u_{0}(x, t)=\sin x \sin t \\
u_{n}(x, t)=0, \forall n \geq 1
\end{array}\right.
$$

and the exact solution of Equation (64) is

$$
u(x, t)=\sin x \sin t
$$

\subsection{Problem 2}

Consider the following nonlinear Klein-Gordon equation

$$
\left\{\begin{array}{l}
\frac{\partial^{2} u(x, t)}{\partial t^{2}}=\frac{\partial^{2} u(x, t)}{\partial x^{2}}+u(x, t)-u^{2}(x, t)+x t+x^{2} t^{2} \\
u(x, 0)=1 \\
\frac{\partial u(x, 0)}{\partial x}=x
\end{array}\right.
$$

\section{Application of the LADM}

Using the Laplace transform from (73), we have:

$$
\begin{aligned}
\mathcal{L}(u(x, t))= & \frac{s}{s^{2}-1}\left(\frac{1+s^{2}}{s^{2}\left(s^{2}-1\right)}\right) x+\frac{2}{s^{3}\left(s^{2}-1\right)} x^{2} \\
& +\frac{1}{s^{2}-1} \mathcal{L}\left(\frac{\partial^{2} u(x, t)}{\partial x^{2}}\right)-\frac{1}{s^{2}-1} \mathcal{L}\left[u^{2}(x, t)\right]
\end{aligned}
$$

Now, we apply the inverse Laplace transform, we have: 


$$
\begin{aligned}
u(x, t)= & \mathcal{L}^{-1}\left(\frac{s}{s^{2}-1}\right)+\mathcal{L}^{-1}\left(\frac{1+s^{2}}{s^{2}\left(s^{2}-1\right)}\right) x+\mathcal{L}^{-1}\left(\frac{2}{s^{3}\left(s^{2}-1\right)}\right) x^{2} \\
& +\mathcal{L}^{-1}\left(\frac{1}{s^{2}-1} \mathcal{L}\left(\frac{\partial^{2} u(x, t)}{\partial x^{2}}\right)\right)-\mathcal{L}^{-1}\left(\frac{1}{s^{2}-1} \mathcal{L}\left[u^{2}(x, t)\right]\right)
\end{aligned}
$$

Thus

$$
\begin{aligned}
u(x, t)= & 1+x t+\mathrm{e}^{t} x-2 x t-\mathrm{e}^{-t} x+\mathrm{e}^{t} x^{2}+\mathrm{e}^{-t} x^{2}-t^{2} x^{2}-2 x^{2}+\frac{\mathrm{e}^{t}}{2}+\frac{\mathrm{e}^{-t}}{2}-1 \\
& +\mathcal{L}^{-1}\left(\frac{1}{s^{2}-1} \mathcal{L}\left(\frac{\partial^{2} u(x, t)}{\partial x^{2}}\right)\right)-\mathcal{L}^{-1}\left(\frac{1}{s^{2}-1} \mathcal{L}\left[u^{2}(x, t)\right]\right)
\end{aligned}
$$

Denoting that the solution of (73) has the following form:

$$
\begin{gathered}
u(x, t)=\sum_{n=0}^{+\infty} u_{n}(x, t) \\
u^{2}(x, t)=\sum_{n=0}^{+\infty} A_{n}
\end{gathered}
$$

Taking (77) and (78) into (76), we have:

$$
\begin{aligned}
\sum_{n=0}^{+\infty} u_{n}(x, t)= & 1+x t+\mathrm{e}^{t} x-2 x t-\mathrm{e}^{-t} x+\mathrm{e}^{t} x^{2}+e^{-t} x^{2}-t^{2} x^{2}-2 x^{2}+\frac{\mathrm{e}^{t}}{2}+\frac{\mathrm{e}^{-t}}{2}-1 \\
& +\sum_{n=0}^{+\infty}\left(\mathcal{L}^{-1}\left(\frac{1}{s^{2}-1} \mathcal{L}\left(\frac{\partial^{2} u_{n}(x, t)}{\partial x^{2}}\right)\right)-\mathcal{L}^{-1} \mathcal{L}\left(\frac{1}{s^{2}-1}\left(A_{n}\right)\right)\right)
\end{aligned}
$$

and we obtain the following Adomian algorithm:

$$
\left\{\begin{aligned}
u_{0}(x, t)=1+x t & \\
u_{1}(x, t)= & \mathrm{e}^{t} x-2 x t-\mathrm{e}^{-t} x+\mathrm{e}^{t} x^{2}+\mathrm{e}^{-t} x^{2}-t^{2} x^{2}-2 x^{2}+\frac{\mathrm{e}^{t}}{2}+\frac{\mathrm{e}^{-t}}{2}-1 \\
& +\mathcal{L}^{-1}\left(\frac{1}{s^{2}-1} \mathcal{L}\left(\frac{\partial^{2} u_{0}(x, t)}{\partial x^{2}}\right)\right)-\mathcal{L}^{-1}\left(\frac{1}{s^{2}-1} \mathcal{L}\left(A_{0}\right)\right) \\
u_{n+1}(x, t)= & \mathcal{L}^{-1}\left(\frac{1}{s^{2}-1} \mathcal{L}\left(\frac{\partial^{2} u_{n}(x, t)}{\partial x^{2}}\right)\right)-\mathcal{L}^{-1}\left(\frac{1}{s^{2}-1} \mathcal{L}\left(A_{n}\right)\right), \forall n \geq 1
\end{aligned}\right.
$$

Calculation $u_{1}(x, t)$

$$
\left\{\begin{aligned}
A_{0}=N( & \left.u_{0}\right)=u_{0}^{2}(x, t)=1+2 x t+x^{2} t^{2} \\
u_{1}(x, t)= & \mathrm{e}^{t} x-2 x t-\mathrm{e}^{-t} x+\mathrm{e}^{t} x^{2}+\mathrm{e}^{-t} x^{2}-t^{2} x^{2}-2 x^{2}+\frac{\mathrm{e}^{t}}{2}+\frac{\mathrm{e}^{-t}}{2}-1 \\
& +\mathcal{L}^{-1}\left(\frac{1}{s^{2}-1} \mathcal{L}\left(\frac{\partial^{2}}{\partial x^{2}}(1+x t)\right)\right)-\mathcal{L}^{-1}\left(\frac{1}{s^{2}-1} \mathcal{L}\left(1+2 x t+x^{2} t^{2}\right)\right) \\
= & \mathrm{e}^{t} x-x \mathrm{e}^{t}-2 x t+2 t x-x \mathrm{e}^{-t}+x \mathrm{e}^{-t}+\mathrm{e}^{t} x^{2}-\mathrm{e}^{t} x^{2}+\mathrm{e}^{-t} x^{2}-\mathrm{e}^{-t} x^{2} \\
& -2 x^{2}+2 x^{2}+\frac{1}{2} \mathrm{e}^{t}-\frac{1}{2} \mathrm{e}^{t}+\frac{1}{2} \mathrm{e}^{-t}-\frac{1}{2} \mathrm{e}^{-t}+t^{2} x^{2}-t^{2} x^{2}+1-1 \\
= & 0
\end{aligned}\right.
$$


Thus

$$
\left\{\begin{array}{l}
u_{0}(x, t)=1+x t \\
A_{n}=0, \quad \forall n \geq 1 \\
u_{n}(x, t)=0, \forall n \geq 1
\end{array}\right.
$$

So that, the solution $u(x, t)$ is given by:

$$
\begin{aligned}
u(x, t) & =u_{0}(x, t)+u_{1}(x, t)+u_{2}(x, t)+\cdots \\
& =1+x t+0+0+\cdots=1+x t
\end{aligned}
$$

which is the exact solution of the problem.

\subsection{Problem 3}

Consider the following nonlinear Klein-Gordon equation

$$
\left\{\begin{array}{l}
\frac{\partial^{2} u(x, t)}{\partial t^{2}}=\frac{\partial^{2} u(x, t)}{\partial x^{2}}-u(x, t)-u^{2}(x, t)+x^{2} \cos ^{2} t \\
u(x, 0)=x \\
\frac{\partial u(x, 0)}{\partial t}=0
\end{array}\right.
$$

\section{Application of the LADM}

Using the Laplace transform, we have:

$$
\mathcal{L}(u(x, t))=\frac{s}{s^{2}+1} x+\left(\frac{s^{2}+2}{s\left(s^{2}+1\right)\left(s^{2}+4\right)}\right) x^{2}+\frac{1}{\left(s^{2}+1\right)} \mathcal{L}\left(\frac{\partial^{2} u(x, t)}{\partial x^{2}}\right)-\frac{1}{s^{2}+1} \mathcal{L}\left[u^{2}(x, t)\right]
$$

The inverse Laplace transformation is applied to Equation (85) we get

$$
\begin{aligned}
u(x, t)= & x^{-1}\left(\frac{s}{s^{2}+1}\right)+x^{2} \mathcal{L}^{-1}\left(\frac{s^{2}+2}{s\left(s^{2}+1\right)\left(s^{2}+4\right)}\right) \\
& +\mathcal{L}^{-1}\left[\frac{1}{\left(s^{2}+1\right)} \mathcal{L}\left(\frac{\partial^{2} u(x, t)}{\partial x^{2}}\right)\right]-\mathcal{L}^{-1}\left(\frac{1}{s^{2}+1} \mathcal{L}\left[u^{2}(x, t)\right]\right) \\
= & x \cos t+\frac{1}{2} x^{2}-\frac{1}{6} x^{2} \cos 2 t-\frac{1}{3} x^{2} \cos t \\
& +\mathcal{L}^{-1}\left(\frac{1}{\left(s^{2}+1\right)} \mathcal{L}\left(\frac{\partial^{2} u(x, t)}{\partial x^{2}}\right)\right)-\mathcal{L}^{-1}\left(\frac{1}{s^{2}+1} \mathcal{L}\left[u^{2}(x, t)\right]\right)
\end{aligned}
$$

As before, we defines the solution $u(x, t)$ by the series

$$
u(x, t)=\sum_{n=0}^{+\infty} u_{n}(x, t)
$$

and $u_{x x}$ can be defined by an infinite series

$$
\frac{\partial^{2} u(x, t)}{\partial x^{2}}=\sum_{n=0}^{+\infty} \frac{\partial^{2} u_{n}(x, t)}{\partial x^{2}}
$$

The nonlinear term $N(u)=u^{2}$ is decomposed in term of Adomian polynomials 


$$
N(u(x, t))=\sum_{n=0}^{+\infty} A_{n}
$$

Substituting (87), (88) and (89) into both sides of Equation (86) we obtain

$$
\begin{aligned}
\sum_{n=0}^{+\infty} u_{n}(x, t)= & x \cos t+\frac{1}{2} x^{2}-\frac{1}{6} x^{2} \cos 2 t-\frac{1}{3} x^{2} \cos t \\
& +\sum_{n=0}^{+\infty} \mathcal{L}^{-1}\left(\frac{1}{\left(s^{2}+1\right)} \mathcal{L}\left(\frac{\partial^{2} u_{n}(x, t)}{\partial x^{2}}\right)\right)-\sum_{n=0}^{+\infty} \mathcal{L}^{-1}\left(\frac{1}{s^{2}+1} \mathcal{L}\left(A_{n}\right)\right)
\end{aligned}
$$

The recursive relation is defined by

$$
\left\{\begin{array}{l}
u_{0}(x, t)=x \cos t \\
u_{1}(x, t)=\frac{1}{2} x^{2}-\frac{1}{6} x^{2} \cos 2 t-\frac{1}{3} x^{2} \cos t+\mathcal{L}^{-1}\left(\frac{1}{\left(s^{2}+1\right)} \mathcal{L}\left(\frac{\partial^{2} u_{0}(x, t)}{\partial x^{2}}\right)\right)-\mathcal{L}^{-1}\left(\frac{1}{s^{2}+1} \mathcal{L}\left(A_{0}\right)\right) \\
u_{n+1}(x, t)=\mathcal{L}^{-1}\left(\frac{1}{\left(s^{2}+1\right)} \mathcal{L}\left(\frac{\partial^{2} u_{n}(x, t)}{\partial x^{2}}\right)\right)-\mathcal{L}^{-1}\left(\frac{1}{s^{2}+1} \mathcal{L}\left(A_{n}\right)\right) ; \forall n \geq 1
\end{array}\right.
$$

(91) give us

$$
\left\{\begin{aligned}
A_{0}=u_{0}^{2}= & x^{2} \cos ^{2} t \\
u_{1}(x, t)= & \frac{1}{2} x^{2}-\frac{1}{6} x^{2} \cos 2 t-\frac{1}{3} x^{2} \cos t \\
& +\mathcal{L}^{-1}\left(\frac{1}{\left(s^{2}+1\right)} \mathcal{L}\left(\frac{\partial^{2}}{\partial x^{2}}(x \cos t)\right)\right)-\mathcal{L}^{-1}\left(\frac{1}{s^{2}+1} \mathcal{L}\left(A_{n}\right)\right) \\
= & \frac{1}{2} x^{2}-\frac{1}{2} x^{2}-\frac{1}{6} x^{2} \cos 2 t+\frac{1}{6} x^{2} \cos 2 t-\frac{1}{3} x^{2} \cos t+\frac{1}{3} x^{2} \cos t \\
= & 0
\end{aligned}\right.
$$

Thus

$$
\left\{\begin{array}{l}
u_{0}(x, t)=x \cos t \\
A_{0}=x^{2} \cos ^{2} t \\
u_{n}(x, t)=0, \forall n \geq 1 \\
A_{n}=0, \forall n \geq 1
\end{array}\right.
$$

and the exact solution of Equation (84) is

$$
u(x, t)=x \cos t
$$

\section{Conclusion}

Through these examplles, we showed again the usefulness of Laplace-Adomian Decomposition method and the He-Laplace method, in the search of an approximate solution of Klein-Gordon equation holds for the accepted forms of strong interaction of antiparticles in modern physics.

\section{References}

[1] Behe, H.A. (2002) Modern Quantum Theory. 4th Edition, Freeman and Co., San Francisco. 
[2] Fadaei, J. (2011) Application of Laplace-Adomian Decomposition Method on Linear and Nonlinear System of PDEs. Applied Mathematical Sciences, 5, 1307-1315.

[3] Abbaoui, K. (1995) Les fondements de la méthode décompositionnelle d'Adomian et application à la résolution de problèmes issus de la biologie et de la médécine. Thèse de doctorat de l'Université Paris VI.

[4] Abbaoui, K. and Cherruault, Y. (1994) Convergence of Adomian Method Applied to Differential Equations. Mathematical and Computer Modellings, 28, 103-109.

[5] Abbaoui, K. and Cherruault, Y. (1994) Convergence of Adomian's Method Applied to Non Linear Equations. Mathematical and Computer Modelling, 20, 60-73.

[6] Abbaoui, K. and Cherruault, Y. (1999) The Decomposition Method Applied to the Cauchy Problem. Kybernetes, 28, 68-74. http://dx.doi.org/10.1108/03684929910253261

[7] He, J.H. (2005) Application of Homotopy Perturbation Method to Nonlinear Wave Equation. Chaos, Solitons, Fractals, 26, 295-300. http://dx.doi.org/10.1016/j.chaos.2005.03.006

Submit or recommend next manuscript to SCIRP and we will provide best service for you:

Accepting pre-submission inquiries through Email, Facebook, LinkedIn, Twitter, etc. A wide selection of journals (inclusive of 9 subjects, more than 200 journals)

Providing 24-hour high-quality service

User-friendly online submission system

Fair and swift peer-review system

Efficient typesetting and proofreading procedure

Display of the result of downloads and visits, as well as the number of cited articles

Maximum dissemination of your research work

Submit your manuscript at: http://papersubmission.scirp.org/

Or contact jamp@scirp.org 